\title{
Estimulación psicocognoscitiva en las demencias
}

\section{Psychocognitive stimulation in dementias}

\author{
I. Francés, M. Barandiarán, T. Marcellán, L. Moreno
}

\section{RESUMEN}

La enfermedad de Alzheimer es un trastorno neurodegenerativo que provoca una progresiva pérdida de memoria y de otras funciones cognitivas, con síntomas también en la esfera conductual y psicológica, pérdida de autonomía y todo ello con una importante sobrecarga para la familia y el entorno social. El abordaje de este trastorno y de otras demencias afines requiere una estrategia multidimensional que pueda afrontar las diversas necesidades que plantea el enfermo y su familia. En ausencia de un tratamiento farmacológico curativo, a lo largo de los años, han ido surgiendo intervenciones psicososiales dirigidas a optimizar la función del enfermo y apoyar a la familia en su cuidado, técnicas muy diversas en cada uno de los ámbitos de intervención (cognitivo, conductual, ambiente, familiar). En esta revisión, hacemos una descripción de las intervenciones más estudiadas en el campo de la estimulación cognitiva, desde las aproximaciones más globales (terapia de orientación a la realidad, terapia de reminiscencia, programas de psicoestimulación) a las más específicas en el campo de la rehabilitación de la memoria (entrenamiento de memoria explícita, técnica de recuerdo demorado, difuminación, aprendizaje sin error, entrenamiento de memoria procedimental y empleo de ayudas externas), y se muestran los resultados de algunos estudios realizados siguiendo estas técnicas de intervención.

Palabras clave. Enfermedad de Alzheimer. Demencia. Entrenamiento cognitivo. Psicoestimulación. Terapias no farmacológicas.

\begin{abstract}
Alzheimer's disease is a neurodegenerative disorder that provokes a progressive loss of memory and of other cognitive functions, with additional symptoms in the behavioural and psychological sphere, loss of autonomy and an important overburdening for the family and the social milieu. Dealing with this disorder and other related dementias requires a multidimensional strategy that is able to face the different needs raised by the patient and his family. Given the lack of a curative pharmacological treatment, psychosocial interventions have emerged over the years that are directed at optimising the function of the patient and supporting the family in caring for him, with very different techniques in each of the spheres of intervention (cognitive, behavioural, environmental, family). In this review, we make a description of the interventions that have been most studied in the field of cognitive stimulation, from the most global approaches (reality orientation therapy, reminiscence therapy, psychostimulation programs) to the most specific ones in the field of memory rehabilitation (training of the explicit memory, technique of delayed remembrance, vagueness, errorfree learning, procedure training of the memory and the use of external help), and the results are shown of some studies carried out using these techniques of intervention.

Key words. Alzheimer's disease. Dementia. Cognitive training. Cognitive stimulation. Non-
\end{abstract} pharmacological therapies.
Aceptado para su publicación el 8 de octubre de 2003.
Correspondencia

Inés Francés Román

Clínica Psicogeriátrica "Josefina Arregui"

Travesía Zelai, s/n

31800-Alsasua

Tfno: 948-563850

Fax: 948-563961

e-mail: ifrances@worldonline.es 


\section{INTRODUCCIÓN}

La enfermedad de Alzheimer es un trastorno neurodegenerativo que habitualmente comienza con pérdida de memoria, al que se van asociando alteraciones de otras funciones mentales, con una progresiva afectación de las actividades diarias del sujeto conduciéndolo a una situación de dependencia. Con frecuencia, la enfermedad cursa también con síntomas de la esfera conductual y psicológica, síntomas que generan una importante carga para los familiares y cuidadores, y para la sociedad, por ser los determinantes de la institucionalización en la mayoría de los casos.

$\mathrm{Al}$ igual que la enfermedad de Alzheimer, otras demencias como la demencia vascular, la demencia frontal, la enfermedad por cuerpos de Lewy, por citar las más frecuentas, van a cursar con deterioro de la función cognitiva, aparición de dependencia funcional, alteraciones del comportamiento más o menos graves y una progresiva necesidad de cuidados por parte del entorno (familiares y cuidadores).

En la última década, fármacos como los inhibidores de la acetilcolinesterasa han demostrado eficacia en el control temporal de los síntomas cognitivos, conductuales y funcionales de la enfermedad de Alzheimer. Pero en ausencia de un tratamiento curativo se hace necesario un abordaje terapéutico multidimensional que incluya, además de las intervenciones farmacológicas, intervenciones no farmacológicas dirigidas a optimizar la cognición, la conducta y la función de los sujetos con demencia, y que además atienda las necesidades de los cuidadores.

Este tipo de intervenciones se viene aplicando desde hace décadas, aunque no siempre con una base sólida. De hecho, la mayoría de las intervenciones en el cuidado de los sujetos con demencia no están basadas en una técnica sistematizada o estructurada; la mayoría de los trabajadores que cuidan de estos enfermos no reconocen que los cuidados ambientales que ellos proporcionan, así como sus interacciones constituyan de hecho una intervención ${ }^{1}$.

El modelo en el que se basan las intervenciones psicosociales en las demencias es en la noción de "exceso de discapaci- dad". Las personas pueden mostrar un mayor grado de discapacidad que aquél que correspondería por los cambios neuropatológicos del cerebro. La teoría de Kitwood sobre los cuidados en demencia sugiere que un entorno social invalidante y deshumanizante interacciona con la fisiopatología cerebral para producir la conducta y la función observadas en las personas con demencia. Kitwood ${ }^{2}$ argumenta que este entorno puede incrementar la progresión de los cambios neuropatológicos formando una espiral de declinar $\mathrm{y}$ degeneración.

Por ello, todas estas intervenciones dirigidas al cuidado de personas con demencia están destinadas a mantener y estimular las capacidades preservadas del individuo, intentando conseguir la mejor situación funcional posible en cada estadio de la enfermedad y con ello ralentizar el declive que pudiera venir generado por factores externos.

A pesar del trabajo que se está realizando en este campo y la actividad clínica habitual en la que se desarrollan diferentes programas de intervención (psicosociales, cognitivos, etc.) existe poca evidencia sobre la eficacia real de los mismos. Se está realizando un esfuerzo importante para aplicar la metodología de los ensayos clínicos a las intervenciones psicosociales, pero se topa con importantes problemas metodológicos: a) La propia naturaleza de la enfermedad, progresiva y clínicamente heterogénea condiciona la aplicación de los diferentes programas y la evaluación de su eficacia. b) La dificultad de estandarizar y comparar resultados con protocolos de intervención grupal, que sean al mismo tiempo individualizados. c) La elección de parámetros de eficacia adecuados, capaces de medir el efecto de la intervención, no sólo en el área cognitiva, sino también conductual y funcional. Son estos aspectos funcionales y el impacto sobre la calidad de vida del paciente y de los cuidadores, los que mejor pueden demostrar el beneficio real del tratamiento. d) Las dificultades en el diseño de estudios multicéntricos, aleatorizados con un grupo control y ciegos, con un número suficiente de pacientes que permita obtener conclusiones acerca de la eficacia del método empleado. 
Por este motivo, la mayor parte de los estudios que intentan evaluar las diferentes intervenciones propuestas están realizados con muestras pequeñas. Por otra parte, dada la heterogeneidad clínica y el amplio rango de severidad de las demencias, se acepta la necesidad de intervenciones altamente individualizadas, diseñadas en función de las necesidades de los pacientes, argumento que apoya la necesidad de estudios de caso-único bien diseñados, sin que esta metodología deba ser rechazada a favor de los ensayos controlados ${ }^{1}$.

Son muchas las intervenciones psicosociales propuestas para el tratamiento de los pacientes con demencia: unas enfocadas al entrenamiento de funciones cognitivas, otras al tratamiento de los problemas de conducta, otras dirigidas específicamente a disminuir la dependen- cia del paciente a través de técnicas de reestructuración ambiental y otras enfocadas al núcleo familiar (Fig.1). Está claro que la intervención en cualquiera de estas áreas puede influir sobre las otras, generalmente de forma positiva, pero también puede tener un efecto negativo, de ahí la importancia de fijar claramente los objetivos a conseguir con cada intervención y evaluar su repercusión en todas las áreas mencionadas. La investigación irá definiendo cuáles de estas intervenciones aportan más beneficios a los pacientes y a sus familias.

En la presente revisión sólo vamos a hacer referencia a las intervenciones dirigidas específicamente a la mejora o mantenimiento de la función cognitiva, bien de forma global o bien en áreas específicas, como la memoria (Tabla 1).

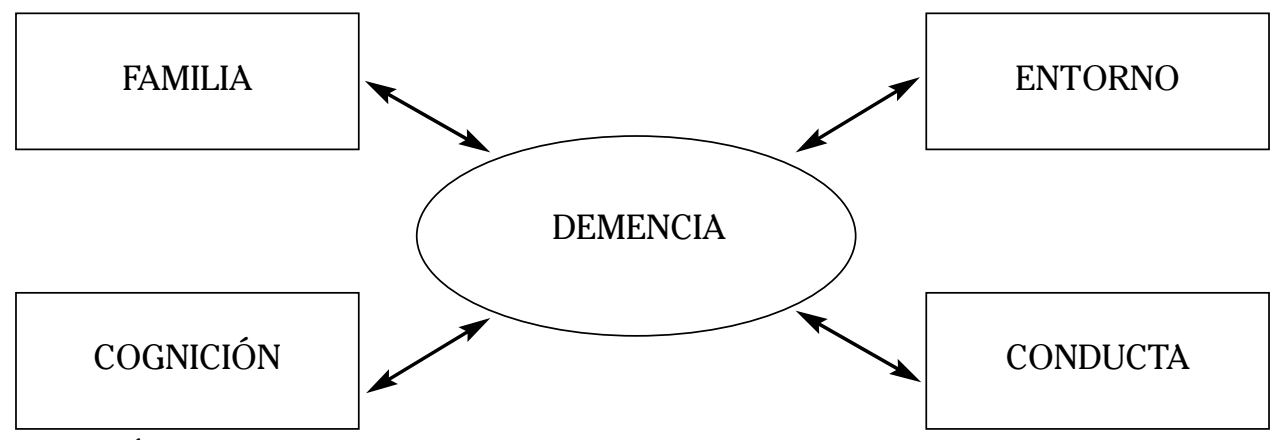

Figura 1. Ámbitos de intervención en la demencia a través de terapias no farmacológicas ${ }^{59}$.

Tabla 1. Clasificación de los tratamientos no farmacológicos en las demencias ${ }^{59}$.

1. Programas de estimulación y actividad.

- Terapia de orientación a la realidad

- Reminiscencia

- Musicoterapia

- Actividad física

- Programa de psicoestimulación integral

2. Reestructuración ambiental.

3. Técnicas de modificación de conducta.

4. Programas para familiares.

5. Entrenamiento en capacidades cognitivas específicas.

6. Nuevas intervenciones.

- Programas interactivos con ordenadores

- Programa intergeneracional (metodología Montessori) 


\section{PROGRAMAS DE ESTIMULACIÓN Y ACTIVIDAD}

Éstos son los programas con más tradición dentro de las terapias no farmacológicas. Se basan en la creencia de que mantener a la persona con demencia, activa y estimulada, tanto desde el punto de vista intelectual como físico, puede disminuir o ralentizar el declive cognitivo y funcional, estimulando aquellas áreas que todavía están preservadas y evitando el desuso que puede venir derivado de la falta de competencia en otras funciones. Esta teoría vendría avalada por recientes estudios que demuestran cómo la participación frecuente en actividades que estimulan la cognición (leer, jugar a las cartas o al ajedrez, visitar museos, escuchar música o tocar algún instrumento, hacer crucigramas, ver la televisión, etc.) puede disminuir el riesgo de enfermedad de Alzheimer ${ }^{3}$ y de demencia ${ }^{4}$ en personas ancianas. Sería necesario estudiar cómo la participación en estas actividades influye sobre la enfermedad una vez instaurado el declive cognitivo.

\section{Terapia de orientación a la realidad}

La orientación a la realidad (OR) fue descrita por primera vez por Folsom ${ }^{5}$ como una técnica para mejorar la calidad de vida de personas ancianas con estados de confusión, aunque sus orígenes se remontan a los intentos por rehabilitar a veteranos de guerra seriamente trastornados y no al ámbito del trabajo geriátrico. Opera mediante la presentación de información relacionada con la orientación (tiempo, espacio y persona), lo cual se considera que proporciona a la persona una mayor comprensión de aquello que le rodea, y posiblemente produce un aumento en la sensación de control y en la autoestima. Antes de ella, había pocos estudios acerca del uso de terapias psicológicas para la demencia. En ese momento, la orientación a la realidad fue considerada por muchos como un hito que marcó el inicio de las intervenciones psicológicas en la atención en demencia.

Se ha venido aplicando de dos formas diferentes: a) La Orientación a la Realidad en sesiones de entre 30 y 60 minutos de duración, donde se trabaja en pequeños grupos de pacientes, generalmente varias veces a la semana. Las sesiones tienen un enfoque cognitivo, comenzando habitualmente por la orientación temporal, espacial y siguiendo por la discusión de eventos, generalmente del entorno próximo a los pacientes. Como instrumentos de trabajo se utilizó el clásico Tablero de Orientación que típicamente presenta información como el día, la fecha, la estación del año, el nombre del lugar y otras informaciones adicionales. Se utilizan además periódicos, cuadernos de notas o diarios personales y objetos o pinturas que muestren el objeto de la discusión.

b) Otra variedad es la Orientación a la Realidad de 24 horas, en la que la reorientación está presente durante todo el día y es practicada por todas las personas que toman contacto con el paciente a través de referencias en el ambiente, señales y otras ayudas para la memoria. Originalmente, el énfasis estaba centrado en administrar al paciente información a cualquier oportunidad. Más recientemente, se ha desarrollado una aproximación más ecológica en la que el staff responde directamente a las preguntas o dudas de los pacientes o les ayuda a descubrir las respuestas por ellos mismos $^{6,7}$. Esta variante probablemente tenga menos riesgo de sobreestimulación para el paciente que cuando se le proporciona información que no es capaz de asimilar.

La OR ha sido la terapia más ampliamente evaluada. El primer ensayo randomizado fue el publicado por Brook y col${ }^{8}$, en el que comparaba un grupo de pacientes que asistían a sesiones de orientación a la realidad y otro que acudía a una actividad no estructurada en una habitación especialmente equipada con el material de reorientación, estudio que muestra un efecto positivo de las sesiones sobre la función de los pacientes, evaluado por el staff.

Una revisión sistemática realizada por Spector y $\mathrm{col}^{9}$, en el año 2000 , y publicada en la Cochrane Library examina la evidencia acerca de la efectividad de las sesiones 
de Orientación a la Realidad en personas con demencia. En esta revisión se incluyeron todos los estudios controlados aleatorizados encontrados tras una amplia búsqueda bibliográfica, que aseguraran un periodo de estudio mínimo de tres semanas con un número mínimo de 10 sesiones. De los 21 estudios controlados encontrados, sólo 8 eran aleatorios y de esos ocho estudios sólo seis pudieron ser introducidos en el metanálisis, ya que los otros dos no proporcionaban resultados necesarios para el mismo. Entre los 6 ensayos, hubo un total de 125 sujetos (67 en grupo experimental y 58 en grupo control) (Tabla 2).

Los resultados mostraron un efecto positivo a favor del tratamiento tanto en el área cognitiva como en la conductual, aunque no llegaron a tener significación estadística. Sólo un estudio, el de Breuil y $\mathrm{col}^{17}$ mostró resultados estadísticamente significativos en la esfera cognitiva a favor de la

Tabla 2. Descripción metodológica de los estudios incluidos en la revisión de la Cochrane Library sobre la Terapia de Orientación a la Realidad en demencia. (Fuente: revisión de la Cochrane Library, 2000').

\begin{tabular}{|c|c|c|c|c|c|c|}
\hline & $\begin{array}{l}\text { Pacientes } \\
\text { (criterios de } \\
\text { inclusión) }\end{array}$ & $\begin{array}{l}\text { Duración de las } \\
\text { sesiones/ } \\
\text { n⿳o sesiones/ }^{0} \text { duración del } \\
\text { tratamiento }\end{array}$ & $\begin{array}{l}\text { Actividades } \\
\text { durante la } \\
\text { Orientación a } \\
\text { la Realidad }\end{array}$ & $\begin{array}{l}\text { Actividades } \\
\text { grupo control }\end{array}$ & $\begin{array}{l}\text { Escalas } \\
\text { cognitivas }\end{array}$ & $\begin{array}{l}\text { Escalas } \\
\text { conductuales }\end{array}$ \\
\hline $\begin{array}{l}\text { Woods }^{10} \\
\text { (1979) }\end{array}$ & $\begin{array}{l}\text { Cociente de } \\
\text { memoria } \leq 70 \\
\text { en la Weschler } \\
\text { Memory Scale. } \\
\end{array}$ & $\begin{array}{l}30 \text { minutos } \\
5 \text { veces/sem. } \\
20 \text { semanas }\end{array}$ & $\begin{array}{l}\text { Tablero de OR, } \\
\text { orientación, } \\
\text { discusiones, } \\
\text { demostraciones. }\end{array}$ & Terapia social & $\begin{array}{l}\text { Wechsler } \\
\text { Information/ } \\
\text { Orientation/ } \\
\text { Concentration }\end{array}$ & - \\
\hline $\begin{array}{l}\text { Hanley col }^{11} \\
\text { (1981) }\end{array}$ & $\begin{array}{l}\text { Demencia entre } \\
\text { leve y grave } \\
\text { (según el test de } \\
\text { Koskela) }\end{array}$ & $\begin{array}{l}30 \text { min } \\
4 \text { veces/sem. } \\
12 \text { semanas }\end{array}$ & $\begin{array}{l}\text { Tablero de OR, } \\
\text { relojes, } \\
\text { calendarios, } \\
\text { mapas. }\end{array}$ & $\begin{array}{l}\text { Ningún } \\
\text { tratamiento. }\end{array}$ & $\begin{array}{l}\text { Koskela Test } \\
\text { Orientation } \\
\text { test }\end{array}$ & $\begin{array}{l}\text { Geriatric } \\
\text { Rating Scale }\end{array}$ \\
\hline $\begin{array}{l}\text { Wallis y col }{ }^{12} \\
\text { (1983) }\end{array}$ & $\begin{array}{l}\text { Sujetos con } \\
\text { demencia o } \\
\text { trastornos } \\
\text { psiquiátricos } \\
\text { funcionales en } \\
\text { régimen de } \\
\text { larga estancia }\end{array}$ & $\begin{array}{l}30 \text { min } \\
5 \text { veces/sem. } \\
3 \text { meses }\end{array}$ & $\begin{array}{l}\text { Tablero de OR, } \\
\text { repetición de } \\
\text { información de } \\
\text { orientación. }\end{array}$ & $\begin{array}{l}\text { Terapia } \\
\text { ocupacional } \\
\text { recreativa }\end{array}$ & $\begin{array}{l}\text { RCP mental } \\
\text { scale }\end{array}$ & Crichton \\
\hline $\begin{array}{l}\text { Baines y col }{ }^{13} \\
\text { (1987) }\end{array}$ & $\begin{array}{l}\text { Alteración } \\
\text { cognitiva } \\
\text { moderada-severa }\end{array}$ & $\begin{array}{l}30 \text { min. } \\
5 \text { veces/sem. } \\
4 \text { semanas }\end{array}$ & $\begin{array}{l}\text { Tablero de OR. } \\
\text { Marerial para } \\
\text { estimular los } \\
\text { sentidos. }\end{array}$ & $\begin{array}{l}\text { Reminiscencia } \\
\text { o ningún } \\
\text { tratamiento. }\end{array}$ & $\begin{array}{l}\text { Cognitive } \\
\text { Assessmet } \\
\text { Scale of } \\
\text { CAPE }\end{array}$ & $\begin{array}{l}\text { CAPE } \\
\text { Behavioural } \\
\text { Rating Scale }\end{array}$ \\
\hline $\begin{array}{l}\text { Ferrario y col }{ }^{14} \\
\text { (1991) }\end{array}$ & $\begin{array}{l}\text { Ancianos } \\
\text { institrucionalizados } \\
\text { con alteración } \\
\text { cognitiva } \\
\end{array}$ & $\begin{array}{l}60 \text { min. } \\
5 \text { veces/sem. } \\
21 \text { semanas }\end{array}$ & No detalles & $\begin{array}{l}\text { Ningún } \\
\text { tratamiento. }\end{array}$ & $\begin{array}{l}\text { CAS (Clifton } \\
\text { Assessment } \\
\text { Schedule) }\end{array}$ & MOSES \\
\hline $\begin{array}{l}\text { Gerber y col }^{15} \\
\text { (1991) }\end{array}$ & $\begin{array}{l}\text { Demencia } \\
\text { degenerativa } \\
\text { primaria } \\
\text { (criterios DSM-III) }\end{array}$ & $\begin{array}{l}60 \text { min. } \\
4 \text { veces/sem. } \\
12 \text { semanas }\end{array}$ & $\begin{array}{l}\text { Tablero de OR, } \\
\text { ejercicios, } \\
\text { preparación de } \\
\text { alimentos, } \\
\text { discusiones. }\end{array}$ & $\begin{array}{l}\text { Interacción } \\
\text { social o } \\
\text { atención } \\
\text { hospitalaria } \\
\text { habitual }\end{array}$ & $\begin{array}{l}\text { Kingston } \\
\text { Dementia } \\
\text { Rating Scale }\end{array}$ & - \\
\hline $\begin{array}{l}\text { Baldelli y col }^{16} \\
\text { (1993) }\end{array}$ & $\begin{array}{l}\text { Demencia senil } \\
\text { tipo Alzheimer }\end{array}$ & $\begin{array}{l}60 \text { min. } \\
3 \text { veces/sem. } \\
3 \text { meses }\end{array}$ & No detalles & $\begin{array}{l}\text { Ningún } \\
\text { tratamiento. }\end{array}$ & MMSE & $\mathrm{ADL}$ \\
\hline $\begin{array}{l}\text { Breuil y col }{ }^{17} \\
\text { (1994) }\end{array}$ & $\begin{array}{l}\text { Demencia, } \\
\text { (criterios } \\
\text { DSM-III) }\end{array}$ & $\begin{array}{l}60 \text { min. } \\
2 \text { veces/sem. } \\
5 \text { semanas }\end{array}$ & $\begin{array}{l}\text { Dibujo, asociación } \\
\text { de palabras, } \\
\text { denominación de } \\
\text { objetos y } \\
\text { categorización }\end{array}$ & $\begin{array}{l}\text { Ningún } \\
\text { tratamiento. }\end{array}$ & $\begin{array}{l}\text { MMS } \\
\text { Lista de } \\
\text { palabras } \\
\text { (CERAD) } \\
\text { Fluencia } \\
\text { verbal GDS }\end{array}$ & - \\
\hline
\end{tabular}

Ninguno de los ensayos incluidos adoptó el formato de OR administrado durante 24 horas además de las sesiones en el aula. 
intervención. Los trabajos aportaron pocos datos sobre la repercusión funcional (Tabla 3).

Todos los estudios de esta revisión fueron realizados en residencias u hospitales psicogeriátricos, excepto el de Breuil y $\mathrm{col}^{17}$, que incluyó sujetos que vivían en la comunidad y acudían dos veces por semana a las sesiones de orientación.

Como se puede apreciar en la tabla 1 los ensayos variaban enormemente en distintos factores como la duración de la intervención, la calidad metodológica y las medidas de resultado. Por ejemplo, el estudio de Breuil (1994), difiere en la metodología de intervención utilizada con el grupo de experimentación: no emplea únicamente las técnicas clásicas de la OR sino que se fundamenta en un método de estimulación cerebral global ${ }^{18}$ que se apoya en las imágenes mentales para estimular la codificación, la consolidación y el recuerdo de información.

En el metanálisis no se objetivó relación entre la duración global de las sesiones y los resultados ni en la esfera cognitiva ni en la comportamental. Tampoco se objetivó influencia alguna de la actividad del grupo control, lo cual sugiere que las cualidades reales de la OR y no solamente su simple efecto terapéutico de contacto social y atención, pueden tener un efecto positivo sobre la cognición. No obstante, el equipo podría haber tenido mayores expectativas con el grupo de OR, lo que pudo haber afectado su ejecución.

Los criterios de inclusión también fueron diferentes y entre ellos el grado de severidad de la demencia de los participantes, lo que puede influir en la magnitud de los resultados obtenidos.

Tabla 3. Características de los pacientes y resultados de los estudios incluidos en la revisión de la Cochrane Library sobre la Terapia de Orientación a la Realidad en demencia. (Fuente: revisión de la Cochrane Library, $2000^{\circ}$ ).

\begin{tabular}{|c|c|c|c|c|c|}
\hline & Pacientes & № participantes & Edad media & $\begin{array}{c}\text { Cognición } \\
\text { DEP* } \\
\text { (Intervalo de } \\
\text { confianza del } \\
95 \%) \\
\end{array}$ & $\begin{array}{c}\text { Conducta } \\
\text { DEP* } \\
\text { (Intervalo de } \\
\text { confianza del } \\
95 \% \text { ) } \\
\end{array}$ \\
\hline Woods $^{10}(1979)$ & $\begin{array}{l}\text { Cociente de } \\
\text { memoria } \leq 70 \text { en la } \\
\text { Weschler Memory Scale. }\end{array}$ & 18 & 76,6 & $\begin{array}{c}-0,664 \\
(-2,041,0,713)\end{array}$ & - \\
\hline $\begin{array}{l}\text { Hanley y col } \\
(1981)^{* *}\end{array}$ & $\begin{array}{l}\text { Demencia entre leve y } \\
\text { grave según el test de } \\
\text { Koskela }\end{array}$ & 57 & 79,5 & & \\
\hline $\begin{array}{l}\text { Wallis y col }^{12} \\
\text { (1983) }\end{array}$ & $\begin{array}{l}\text { Sujetos con demencia o } \\
\text { aislamiento en régimen } \\
\text { de larga estancia }\end{array}$ & $\begin{array}{c}38(19 \\
\text { pacientes } \\
\text { orgánicos y } 19 \\
\text { funcionales) }\end{array}$ & 69,9 & $\begin{array}{c}-0,025 \\
(-0,925,0,876)\end{array}$ & $\begin{array}{c}-0,451 \\
(-1,366,0,464)\end{array}$ \\
\hline $\begin{array}{l}\text { Baines y col }{ }^{13} \\
(1987)\end{array}$ & $\begin{array}{l}\text { Alteración cognitiva } \\
\text { moderada-severa }\end{array}$ & 15 & 80,9 & $\begin{array}{c}-0,812 \\
(-1,426,1,061)\end{array}$ & $\begin{array}{c}-1,324 \\
(-2,770,0,123)\end{array}$ \\
\hline $\begin{array}{l}\text { Ferrario y col }{ }^{14} \\
\text { (1991) }\end{array}$ & $\begin{array}{l}\text { Ancianos } \\
\text { institucionalizados } \\
\text { con alteración cognitiva }\end{array}$ & 13 & 82,5 & $\begin{array}{c}-0,962 \\
(-1,989,0,064)\end{array}$ & $\begin{array}{c}-0,591 \\
(-1,581,0,399)\end{array}$ \\
\hline $\begin{array}{l}\text { Gerber y col }^{15} \\
(1991)\end{array}$ & DDP según DSM-III & 19 & 76,5 & $\begin{array}{c}-0,758 \\
(-1,963,0,448)\end{array}$ & - \\
\hline $\begin{array}{l}\text { Baldelli y col }^{16} \\
(1993)^{* *}\end{array}$ & DSTA & 23 (100\% mujeres) & 84,5 & & \\
\hline $\begin{array}{l}\text { Breuil y col }^{17} \\
(1994)\end{array}$ & Demencia, según DSM-III & 56 & $\begin{array}{c}76,1 \text { (g. estudio) } \\
78,3 \text { (control) }\end{array}$ & $\begin{array}{c}-0,714 \\
(-1,256,-0,172)\end{array}$ & - \\
\hline
\end{tabular}

*Los análisis se ajustaron al modelo de efectos aleatorios debido a la heterogeneidad de los ensayos y se utilizaron diferencias estandarizadas de promedios (DEP) porque los ensayos aplicaron diferentes pruebas para medir los mismos resultados En la esfera cognitiva la DEP global fue de $-0,586$ (intervalo de confianza del $95 \%$ : -0,952, $-0,220$ ). La DEP total en el área conductual fue de $-0,659$ (intervalo de confianza del $95 \%$ : $-1,268 ;-0,050$ ).

**Estos dos estudios no se incluyeron en el metanálisis por falta de datos. 
Los estudios tampoco proporcionan evidencias acerca de los beneficios a largo plazo de la OR. Sólo dos de ellos realizaron un seguimiento a largo plazo y sus resultados son contradictorios: Gerber y $\mathrm{col}^{15}$ muestran un peor desempeño a las 10 semanas de seguimiento que antes del tratamiento, mientras que Wallis y col $^{16}$ encontraron que los sujetos tenían puntuaciones más altas en las pruebas cognoscitivas y de conducta un mes después de interrumpida la intervención.

Estudios recientes sí muestran cómo el mantenimiento de la intervención en el tiempo puede proporcionar más beneficios. Zanetti y col ${ }^{19}$ demostraron que personas con enfermedad de Alzheimer moderada sometidos a ciclos repetidos de OR (una media de 15,48 semanas) mostraban un menor declinar cognitivo al cabo de un año de seguimiento comparados con aquellos que habían asistido sólo a un ciclo de OR durante cuatro semanas.

Los trabajos de la revisión Cochrane no mostraron efectos secundarios. Sin embargo hay autores que señalan que si no es aplicada con la suficiente sensibilidad puede conducir a la frustración y a un aumento del estrés del paciente ${ }^{20}$. Han surgido diversas críticas sobre la orientación a la realidad en la práctica clínica y cierto temor de que haya sido aplicada de una forma mecánica sin tener en cuenta las necesidades del individuo ${ }^{21}$. De hecho la OR como programa terapéutico exclusivo, raramente se encuentra en la práctica actual, ya que tiende a confrontar los errores del paciente y no obtiene sustanciales beneficios en la práctica clínica. A pesar de ello, algunos de sus principios e instrumentos (tableros de OR, por ejemplo) sí se utilizan de forma sistemática en la práctica clínica convencional.

\section{Terapia de reminiscencia}

La terapia de reminiscencia (TR) proviene de los primeros trabajos de Butler ${ }^{22}$ en 1963 acerca de la "Revisión de la Vida". Este autor la describe como un proceso mental que ocurre de manera natural, en el cual se traen a la conciencia las experiencias pasadas y los conflictos sin resolver. La TR como concepto psicoanalítico, fue utilizada como un componente del cuidado ocupacional en instituciones de larga estancia para personas mayores. Su objetivo era ayudar a las personas ancianas a colocar sus experiencias en perspectiva y ayudarles a prepararse para la muerte.

Debido a que la memoria remota es habitualmente lo último que se deteriora, se pensaba que la reminiscencia podía ser un medio efectivo para comunicarse con personas con alteraciones mnésicas, centrándose en una habilidad que a menudo continúa estando comparativamente intacta hasta fases más avanzadas en el proceso de la enfermedad. Así el proceso de reminiscencia fue desarrollado como una técnica terapéutica definida por Woods y col ${ }^{23}$ como "un recuerdo verbal o silencioso de sucesos de la vida de una persona, ya sea sola, o con otra persona o grupo de personas". El trabajo frecuentemente incluye reuniones de grupo, por lo menos una vez a la semana, en donde se estimula a los participantes a que hablen acerca de eventos pasados, a menudo con ayudas como fotografías, música, objetos y vídeos del pasado.

Sin embargo, es importante distinguir la terapia de revisión de vida la cual involucra la evaluación de la memoria personal con un terapeuta como oyente, usualmente en una sesión cara a cara, de una reminiscencia más general en la que se realizan discusiones en el seno de un grupo y la cual puede tener como objetivo mejorar la interacción en un ambiente agradable y de forma atractiva. Esta última es la técnica terapéutica habitualmente utilizada en el contexto de la estimulación cognitiva.

Se han realizado estudios acerca de los efectos de la TR en poblaciones sin demencia, pero el primer estudio desarrollado con personas ancianas con demencia fue el de Kiernat ${ }^{24}$. Aunque era un estudio no controlado que utilizaba evaluaciones subjetivas, Kiernat concluyó que "puede estimularse la conversación, despertarse la atención y aumentar el lapso de tiempo de atención". Este autor introdujo la idea de utilizar la TR en personas con demencia. Hasta entonces, muchos habían creído que las personas debían tener cierto grado de memoria y de comprensión para bene- 
ficiarse de ella. Desde 1979, se han publicado varios estudios sobre la aplicación de la TR en pacientes con demencia, sin embargo, solamente parecen haberse realizado tres ensayos controlados aleatorios (Baines y col ${ }^{13}$, Goldwasser y $\mathrm{col}^{25}$ y Orten y $\operatorname{col}^{26}$ ) que son los evaluados por la revisión Cochrane ${ }^{27}$ (Tabla 4).

De ellos sólo el estudio de Baines pudo ser introducido en el metanálisis. Los resultados mostraron una leve mejoría a favor del tratamiento en la esfera conductual, pero los datos son muy limitados para extraer cualquier tipo de conclusión. Existen estudios que muestran efectos positivos de esta técnica sobre la participación y la socialización de los pacientes, y otros señalan cambios en la actitud de los miembros del equipo, factores que posiblemente sea necesario tener en cuenta en un futuro al evaluar este tipo de intervención.

\section{Programas de psicoestimulación}

Las críticas realizadas sobre la Terapia de Orientación a la Realidad y la Terapia de Reminiscencia es que han sido aplicadas de forma genérica, asumiendo que los pacientes sufrían alteraciones cognitivas similares y que, consecuentemente, podían beneficiarse de programas generales de rehabilitación. Aunque esta crítica está bien fundamentada, es importante analizar estas intervenciones en el contexto en el que surgieron y fueron aplicadas: residencias de ancianos u hospitales psicogeriá- tricos en los que los niveles de estímulo posiblemente fueran pobres, en pacientes en estadios moderados-severos de evolución y con estados de confusión superpuestos. Conforme se ha ido avanzando en el conocimiento de la enfermedad y en el diagnóstico precoz, las terapias no farmacológicas salen fuera del ámbito puramente residencial y aparece la necesidad de su aplicación a pacientes que viven en la comunidad, generalmente con un menor grado de deterioro y con otras necesidades de cuidados. De ahí, la aparición de programas más específicos de estimulación que combinan actividades de grupo con otras más individualizadas diseñadas en función de los déficit que presente el enfermo. Estos programas se vienen aplicando fundamentalmente en los centros de día psicogeriátricos y en los centros residenciales, pero se están comenzando a utilizar de forma ambulatoria.

Un ejemplo es el programa desarrollado por Tárraga ${ }^{28}$ al que denomina Programa de Psicoestimulación Integral (PPI). Parte de una visión globalizadora y ecológica de la persona con demencia y se fundamenta en la neuroplasticidad, en la aplicación práctica de la neuropsicología cognitiva y en la aplicación de las técnicas de modificación de conducta. El modelo teórico en el que se asienta es el propuesto por Reisberg y $\mathrm{col}^{29}$, por el cual la persona con enfermedad de Alzheimer sigue un patrón involutivo inverso a la forma-

Tabla 4. Características de los estudios incluidos en la revisión de la Cochrane Library sobre la Terapia de Reminiscencia en la demencia. (Fuente: revisión de la Cochrane Library, 200027).

\begin{tabular}{|c|c|c|c|c|c|c|}
\hline & $\begin{array}{l}\text { Pacientes } \\
\text { (criterios de } \\
\text { inclusión) }\end{array}$ & № pacientes & $\begin{array}{l}\text { Duración de } \\
\text { las sesiones/ } \\
\text { no sesiones/ } \text { duración del } \\
\text { tratamiento }\end{array}$ & $\begin{array}{l}\text { Actividades } \\
\text { grupo control }\end{array}$ & $\begin{array}{l}\text { Escalas } \\
\text { cognitivas }\end{array}$ & $\begin{array}{l}\text { Escalas } \\
\text { conductuales }\end{array}$ \\
\hline $\begin{array}{l}\text { Baines y col }{ }^{13} \\
(1987)\end{array}$ & $\begin{array}{l}\text { Deterioro cognitivo } \\
\text { moderado o severo }\end{array}$ & $\begin{array}{l}15 \\
\text { (Edad media: } \\
81,5 \text { años) }\end{array}$ & $\begin{array}{l}30 \text { min. } \\
5 \text { veces/sem } \\
4 \text { semanas }\end{array}$ & $\begin{array}{l}\text { - Orientación a la } \\
\text { realidad } \\
\text { - Sin tratamiento }\end{array}$ & $\begin{array}{l}\text { Information/ } \\
\text { Orientation } \\
\text { (CAPE) }\end{array}$ & $\begin{array}{l}\text { Behaviour } \\
\text { (CAPE) }\end{array}$ \\
\hline $\begin{array}{l}\text { Goldwasser } \\
\text { y col }^{\mathbf{2 5}} \\
(1987)\end{array}$ & $\begin{array}{l}\text { Diagnóstico clínico } \\
\text { de Demencia }\end{array}$ & $\begin{array}{l}30 \\
\text { (Edad media: } \\
82,3 \text { años) }\end{array}$ & $\begin{array}{l}30 \text { min. } \\
2 \text { veces/sem } \\
5 \text { semanas }\end{array}$ & $\begin{array}{l}\text { - Apoyo social } \\
\text { - Sin tratamiento }\end{array}$ & MMS & $\begin{array}{l}\text { Beck } \\
\text { Depresión } \\
\text { Inventory } \\
\text { Katz-ADL }\end{array}$ \\
\hline $\begin{array}{l}\text { Orten }^{*} \text { y col } \\
(1989)\end{array}$ & $\begin{array}{l}\text { Pacientes "mo- } \\
\text { deradamente } \\
\text { confusos" }\end{array}$ & 56 & $\begin{array}{l}45 \text { min. } \\
1 \text { vez/semana } \\
16 \text { semanas }\end{array}$ & - Sin tratamiento & & \\
\hline
\end{tabular}

*Este estudio se excluyó de la revisión de la Cochrane Library por falta de claridad en los criterios diagnósticos de inclusión. 
ción de la inteligencia descrita por Piaget ${ }^{30}$ y que se ha calificado como "retrogénesis". Las personas que participan en este programa acuden durante cinco días a la semana, ocho horas al día, y participan en los siguientes talleres: psicoestimulación cognitiva donde se trabaja la orientación, atención-concentración, memoria, lenguaje, cálculo, praxias y gnosias; taller de reminiscencia; taller de cinesiterapia, psicoexpresión y musicoterapia; taller ocupacional y taller de mantenimiento de las actividades de la vida diaria.

Los resultados obtenidos por este programa en un estudio observacional de un año de duración sobre 121 pacientes que acudían al centro de día, muestran que los pacientes mejoraban sus puntuaciones en el Mini Mental State (MMSE) a los dos meses de iniciada la intervención, manteniendo estas puntuaciones hasta el sexto mes en que empiezan a declinar. $\mathrm{Al}$ año, la media de puntuación en el MMSE todavía se mantenía discretamente por encima de la basal. El estudio no aporta datos sobre parámetros conductuales. La situación funcional medida a través de la escala de Barthel sólo muestra una muy discreta caída de las puntuaciones al año de la intervención (mayor dependencia) ${ }^{31}$.
Otros programas de psicoestimulación cognitiva, aplicados en sesiones semanales, han sido evaluados en estudios controlados. Ermini-Füngschilling y col $^{32}$ realizaron un estudio controlado sobre 38 pacientes con enfermedad de Alzheimer leve: la mitad acudieron semanalmente a sesiones de entrenamiento cognitivo, de 60 minutos de duración, durante un año y la otra mitad sirvió de grupo control. El entrenamiento se efectúo en grupos de 6 ó 7 participantes. Frente a otras tendencias, los autores defienden la intervención grupal en este estadio de la enfermedad, pues la interacción del grupo favorece la competencia social y, a pesar de las necesidades individuales, los patrones de alteración cognitiva pueden ser más similares. En este caso, el entrenamiento cognitivo fue semiestructurado y consistía en ejercicios cognitivos (orientación, cálculo, memoria, lenguaje) y ejercicios de relajación. (Tabla 5).

Al cabo de un año, el grupo de intervención mantenía estables sus puntuaciones en el MMS, mientras que el grupo control sufría un deterioro de las mismas. También mejoró en el grupo con entrenamiento cognitivo las puntuaciones en una escala de depresión (GDS).

Tabla 5. Estructura y contenido de una sesión típica del programa de entrenamiento cognitivo de Ermini-Fünfschilling y $\mathrm{col}^{32}$.

\begin{tabular}{|l|l|c|}
\hline $\begin{array}{l}\text { Tema } \\
\text { Qué }\end{array}$ & $\begin{array}{l}\text { HIELO } \\
\text { Cómo: contenido, medios materiales }\end{array}$ & Tiempo \\
\hline Comienzo & Orientación en el tiempo/cálculos (ejercicio en grupo) & $5^{\prime}$ \\
\hline Introducción al tema & $\begin{array}{l}\text { Se muestran y se pasan cubitos de hielo (estimulación táctil) } \\
\text { (Ejercicio en grupo). }\end{array}$ & $5^{\prime}$ \\
\hline Información & $\begin{array}{l}\text { Se proporcionan datos sobre el hielo: geográficos, físicos, mediante } \\
\text { mapas, libros, etc. }\end{array}$ & $5^{\prime}$ \\
\hline Memoria/vocabulario & $\begin{array}{l}\text { Se cuelgan de una pizarra 10 dibujos de objetos (patines, piqueta } \\
\text { para hielo, etc.). Los pacientes conversan sobre los objetos y los } \\
\text { ordenan según diferentes categorías (herramientas, ropas, etc.) } \\
\text { (Ejercicio de grupo). }\end{array}$ & $10^{\prime}$ \\
\hline Relajación & Ejercicios físicos suaves. & $5^{\prime}$ \\
\hline Concentración & No verbal, completar un dibujo de un cristal de hielo, papel, lápiz. & $5^{\prime}$ \\
\hline Lenguaje & Dar una explicación de un esquema móvil (Ejercicio de grupo). & $5^{\prime}$ \\
\hline Fluencia semántica & $\begin{array}{l}\text { Escribir: asociaciones con la palabra hielo, palabras relacionadas } \\
\text { en orden alfabético o por categorías. }\end{array}$ & $1^{\prime}$ \\
\hline Memoria & Recordar objetos previamente manipulados. & $5^{\prime}$ \\
\hline Relajación final & $\begin{array}{l}\text { Los pacientes buscan en el índice de un libro de canciones un título } \\
\text { dado relacionado con el tema de la semana. Cantan todos juntos. }\end{array}$ & $5^{\prime}$ \\
\hline
\end{tabular}


Nuestro propio grupo ha llevado a cabo un estudio subvencionado por el Departamento de Salud del Gobierno de Navarra $^{33}$, para valorar la eficacia de un programa de psicoestimulación, en el que se entrenan de forma sistemática y estructurada las diferentes áreas cognitivas (orientación, atención, memoria, lenguaje, praxis, gnosias, cálculo). Dicho programa se aplicó a un grupo de 9 pacientes con enfermedad de Alzheimer en estadio leve o moderado (GDS 3 - 4) durante seis meses, en sesiones de una hora de duración, dos veces por semana, y se comparó con un grupo control de 8 pacientes que no recibió ningún tipo de intervención. Ambos grupos seguían tratamiento farmacológico con inhibidores de la acetilcolinesterasa. La designación a los grupos fue aleatoria aunque no ciega por parte del evaluador. Al cabo de seis meses se observó una diferencia estadísticamente significativa entre ambos grupos en la función cognitiva (evaluada a través del ADAS-cog) a favor del grupo de tratamiento. No se observaron diferencias en las pruebas que medían función, conducta y estado afectivo. La sobrecarga del cuidador sí mostró mejores resultados en el grupo experimental tras la intervención, aunque las diferencias no fueron significativas.

En estos momentos, en nuestro país, se están desarrollando estudios con muestras importantes de pacientes, que pueden ofrecer datos valiosos sobre el efecto de diferentes programas de psicoestimulación.

\section{Las nuevas tecnologías como instrumentos para el entrenamiento cognitivo}

Se están desarrollando nuevas técnicas de entrenamiento cognitivo mediante programas interactivos con ordenadores. Los primeros en desarrollar este modelo fueron Hoffman y $\mathrm{col}^{34}$, quienes diseñaron un programa interactivo para personas con enfermedad de Alzheimer en estadios leve o moderado con el objetivo de ayudar al paciente a manejar y controlar el entorno en el que vive. Utilizan fotografías del paciente y su entorno y se simula en el ordenador una tarea cotidiana que tenga relevancia para el enfermo. El ordenador informa de los éxitos o fracasos y da pautas para ejecutar la tarea cuando se solicita. Los resultados de este programa muestran que los enfermos actúan cada vez con mayor rapidez y necesitan menos ayudas para completar una determinada tarea.

En España, Franco y $\mathrm{col}^{35}$ han desarrollado el programa Gradior, un sistema multimedia de evaluación y rehabilitación neuropsicológica por ordenador que permite la realización de programas de entrenamiento y recuperación de funciones cognitivas superiores en personas que presen$\tan$ déficit o deterioro cognitivo. El programa permite diseñar diferentes pruebas que trabajan la atención, la percepción, la memoria, el cálculo y la orientación, y el usuario interactúa con una pantalla táctil y sigue una serie de instrucciones visuales y/o sonoras hasta completar cada una de las tareas cognitivas propuestas. Los propios autores ${ }^{36}$ llevaron a cabo un estudio sobre el beneficio de este programa en pacientes con enfermedad de Alzheimer leve. Para ello compararon un grupo de 54 pacientes al que aplicaron el programa Gradior durante 18 meses frente a un grupo control de 79 pacientes en los que no se realizó ninguna intervención. La designación no fue aleatoria. Tras el periodo de estudio, el grupo experimental mantenía las puntuaciones en el Mini-Examen Cognoscitivo (MEC) mientras que el grupo control sufría un declive, siendo la diferencia estadísticamente significativa. El grupo experimental también mejoró sus puntuaciones respecto a la evaluación inicial en los aspectos emocionales y comportamentales (evaluados a través de la escala ADAS-no cognitivo).

Otro modelo de intervención, propuesto por Ortiz ${ }^{37}$, es el programa de teleterapia cognitiva que consiste en una serie de ejercicios cognitivos y otros que el paciente sigue desde su casa a través de la televisión y con un teclado, guiado desde una unidad central por un profesional que lo adapta a su nivel de deterioro. No se han publicado resultados sobre la eficacia de esta intervención en pacientes con enfermedad de Alzheimer. 


\section{REHABILITACIÓN DE MEMORIA}

El déficit de memoria es el síntoma principal de la enfermedad de Alzheimer, y va a jugar un papel fundamental en la rehabilitación de otros déficit cognitivos y en el impacto sobre las actividades de la vida diaria. Por otra parte es la única área de la cognición donde las intervenciones rehabilitadoras en pacientes con enfermedad de Alzheimer están basadas en principios bien establecidos derivados del campo de la neuropsicología cognitiva y de la neurorrehabilitación en personas con daño cerebral no progresivo.

Las técnicas específicas de rehabilitación de la memoria en la enfermedad de Alzheimer se basan en el principio de que la pérdida de memoria se produce de una forma gradual y su intensidad es muy variable en los estadios tempranos de la enfermedad, afectando inicialmente a la memoria episódica y a la memoria semántica. Ambas pertenecen al sistema de memoria explícita. La memoria implícita hace referencia a la adquisición de conocimientos verbales y no verbales (por ejemplo: conocimiento procedimental) en ausencia de un reconocimiento consciente del contenido y las circustancias en las cuales se produce ese recuerdo. El sistema de memoria implícita se encuentra relativamente preservado hasta estadios más avanzados de la enfermedad ${ }^{38}$. De estos hallazgos se deduce que durante los estadios iniciales de la enfermedad, determinadas funciones mnésicas son suscepti- bles de estimulación y de intervenciones rehabilitadoras.

Como muestra la figura 2, las técnicas de rehabilitación de la memoria pueden ser divididas en tres niveles (modificado de Butters y $\mathrm{col}^{39}$ ).

\section{FACILITACIÓN DE LA MEMORIA EXPLÍCITA RESIDUAL}

El primer nivel de intervención representa el más tradicional en rehabilitación de memoria, basado en la facilitación de la memoria explícita a través de un soporte dual tanto en la codificación (procesamiento del estímulo por asociación) como en la recuperación posterior (recuerdo de la información almacenada). Se trata de ofrecer al paciente consignas que informan sobre la naturaleza del material que se quiere memorizar, sobre la mejor secuencia de aprendizaje y de recuperación o sobre la manera de manipular mentalmente la información. Así para aprender nueva información, se tratará de mejorar la calidad de la codificación (conseguir una huella mnésica más elaborada). La codificación puede ser mejorada a través de estímulos multimodales ${ }^{40}$. Por ejemplo, aprender las palabras manzana y pera pasaría por una codificación léxico-semántica (frutas), práxica (pelarlas), gustativa (comerlas), etc. La evocación de este material requerirá posteriormente la utilización de los indicios utilizados en la codificación (frutas, se pelan...). Pero también

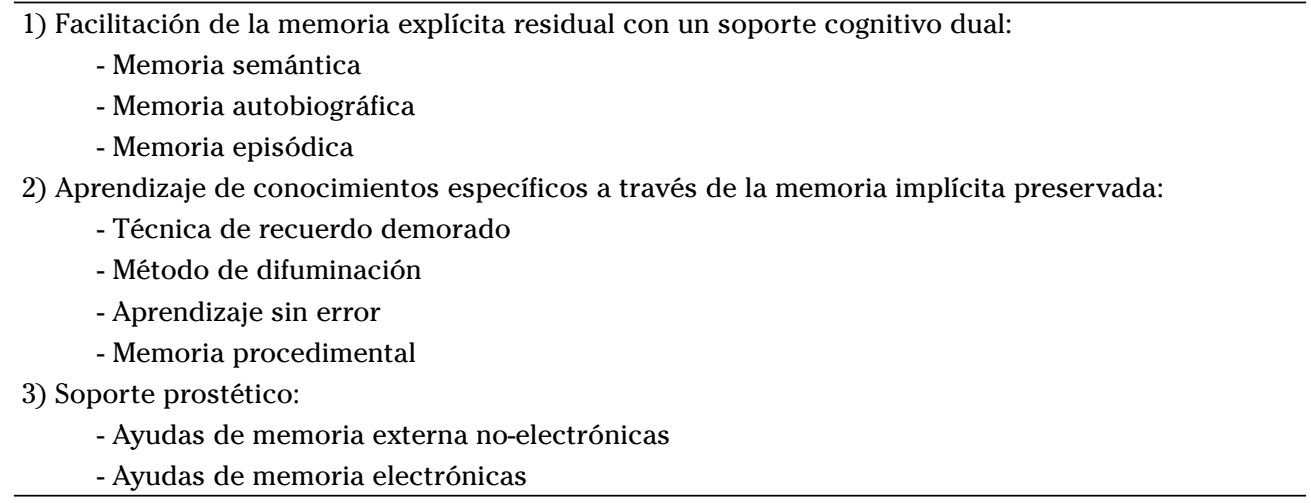

Figura 2. Procedimentos de rehabilitación de la memoria en enfermedad de Alzheimer leve o moderada. (Modificado de Butters y col, 1997) 
otros estímulos pueden mejorar la codificación: la carga emocional ${ }^{41}$, códigos semánticos generados por los propios pacientes $^{42}$, o activando tareas ${ }^{43}$ o eventos relevantes $\operatorname{conocidos}^{44}$ con relación a la información que se quiere aprender.

El significado clínico de estos estudios teóricos ha sido recientemente confirmado con un estudio randomizado ${ }^{45}$ en el que se utilizaba el entrenamiento de memoria para optimizar la memoria episódica residual, semántica y autobiográfica. Veintisiete sujetos con enfermedad de Alzheimer en estadio leve (MMS 20-26) fueron randomizados para recibir placebo (9) o inhibidores de la acetilcolinesterasa (IACE) (18) durante 26 semanas. Después de tres meses de tratamiento, nueve pacientes del grupo de IACE fueron randomizados para recibir un programa individual de entrenamiento de memoria en sesiones de 30-40 minutos dos veces por semana en presencia de un familiar. La intervención consistía en facilitar la codificación (a través de material del propio paciente, actividades motoras, generación de ayudas por el propio paciente) y el recuerdo. Los familiares fueron invitados a repetir los ejercicios en casa entre las sesiones. Al cabo de 26 semanas, las puntuaciones en ADAS-cog mejoraron -6,62 puntos en el grupo de intervención y $-1,27$ en el que sólo recibió IACE, con un deterioro de 4,61 puntos en el grupo control. El porcentaje de pacientes que mejoraron 7 puntos o más en el grupo de intervención fue del $56,6 \%$, del $33,3 \%$ en el que sólo recibió fármacos y del $0 \%$ en el grupo control. También se objetivaron mejorías en las actividades básicas de la vida diaria.

\section{Estimación de la memoria implícita}

El segundo nivel de intervención se refiere al desarrollo de técnicas específicamente diseñadas para el desarrollo de la memoria implícita.

Diferentes estudios sugieren que sería posible hacer aprender a un paciente conocimientos específicos en un determinado campo con el objetivo de ofrecerle más autonomía en su vida cotidiana, y ello gracias a la explotación de sus capacidades de aprendizaje que permanezcan todavía intactas. El objetivo de esta aproximación no es recuperar la capacidad mnésica per se. El objetivo implica, por una parte, delimitar los dominios específicos de conocimiento en los cuales es importante para el paciente adquirir, retener y utilizar nuevos conocimientos, y por otra, desarrollar las técnicas que explotan las capacidades mnésicas preservadas de los pacientes.

Bajo esta perspectiva se utilizan dos métodos para la estimulación psicocognoscitiva:

- el método de recuperación espaciada (spaced retrieval) $^{46}$

Tabla 6. Estudios realizados por Clare y col siguiendo el principio de aprendizaje sin error ${ }^{60.62}$.

\begin{tabular}{|l|l|c|l|l|}
\hline Año & Tipo de estudio & No pacientes & Técnica & Resultados \\
\hline 2000 & Abierto & $\begin{array}{l}6 \text { con E.A. } \\
\text { (MMS: 21-26) }\end{array}$ & $\begin{array}{l}\text { Entrenamiento } \\
\text { de un problema } \\
\text { cotidiano de } \\
\text { memoria }\end{array}$ & $\begin{array}{l}\text { - } 5 \text { de los } 6 \text { pacientes obtuvieron una } \\
\text { mejoría significativa } \\
\text { - La mejoría se mantuvo 6 meses } \\
\text { después de la intervención. }\end{array}$ \\
\hline 2002 & $\begin{array}{l}\text { Controlado sin } \\
\text { grupo control } \\
\text { (MMS } \geq 18)\end{array}$ & $\begin{array}{l}\text { Asociación } \\
\text { cara-nombre }\end{array}$ & $\begin{array}{l}\text { - Mejoría en los resultados de los } \\
\text { ítem entrenados } \\
\text { - No mejoría en los ítem control } \\
\text { - La mejoría se mantuvo 6 meses } \\
\text { después en ausencia de práctica } \\
\text { - Obtuvieron mejores resultados los } \\
\text { pacientes con más conciencia de sus } \\
\text { déficit. }\end{array}$ \\
\hline 2003 & Caso-único & $\begin{array}{l}\text { Mujer de 66 } \\
\text { años en estadio } \\
\text { temprano de } \\
\text { EA }\end{array}$ & $\begin{array}{l}\text { Aprendizaje de 13 } \\
\text { nombres utilizando } \\
\text { una estrategia } \\
\text { nemotécnica }\end{array}$ & $\begin{array}{l}\text { - El recuerdo mejoró del 2,3\% al } \\
\text { 91,46\%. } \\
\text { - Se mantuvo la ganancia durante el } \\
\text { seguimiento. }\end{array}$ \\
\hline
\end{tabular}


- el método de difuminación (vanishing cues $)^{47}$.

a) La técnica de recuperación espaciada consiste en hacer recordar al paciente determinada información en cortos periodos de tiempo que gradualmente se van incrementando $(5,10,20,40,90,120 \mathrm{seg}$, etc.). Si el recuerdo falla en un intervalo, se vuelve al intervalo previo en el que el recuerdo fue acertado, y se continúa tras una reexposición de la información. Si no es capaz de evocar dicha información, el intervalo de tiempo es reducido a la mitad. Los intervalos de tiempo son ocupados por una tarea de interferencia, generalmente algún comentario verbal. Según Camp y collis, el recuerdo correcto después de un intervalo de 15 minutos a una hora, indica que el almacenamiento a largo plazo de la información que se quería aprender se ha realizado con éxito. Algunos trabajos han ido demostrando cómo los pacientes con enfermedad de Alzheimer son capaces de aprender y retener durante varios meses una pequeña cantidad de información importante para sus actividades diarias, por ejemplo recuerdo de la asociación caranombre, localización de objetos, nombres de objetos y orientación. A diferencia de las técnicas de entrenamiento de memoria, ésta puede ser espontáneamente aplicada por el paciente por sí mismo, transfiriéndolo a situaciones fuera del entrenamiento. Sin embargo, y con la excepción de algunos casos no hay evidencia de la generalización espontánea a material no entrenado.

Un estudio controlado y aleatorio realizado con esta técnica por Davis y $\mathrm{col}^{49}$ mostraba una mejoría del recuerdo del material entrenado (información personal y asociación cara-nombre), pero no se apreció una mejoría de los parámetros neuropsicológicos utilizados en la evaluación ni en la calidad de vida del paciente evaluada por los cuidadores.

b) Difuminación. Se trata de una técnica de aprendizaje en que los indicios suministrados al paciente concernientes a la información a recuperar, son difuminados de forma progresiva. Por ejemplo, imaginemos que el paciente debe aprender una asociación nombre/profesión. Una vez presentada la información se le pide que recuerde qué profesión correspondía a un nombre presentado. Si falla, se van añadiendo letras de la profesión a recordar, una a una, hasta que consiga la respuesta correcta. En la siguiente sesión, al paciente se le presenta la respuesta buscada con tantas letras como requirió en el anterior aprendizaje, menos una. Progresivamente las letras irán difuminándose una a una durante el proceso de aprendizaje. Esta técnica se ha utilizado con éxito en pacientes con enfermedad de Alzheimer para el aprendizaje de nombres y profesiones del staff y otros ítem como direcciones y números de teléfono, manteniendo el aprendizaje durante varias semanas. Finalmente, los pacientes han podido extrapolar esos nuevos conocimientos adquiridos a nuevos contextos. No queda claro si este método opera por reforzamiento de la memoria implícita solo o también a través de la memoria explícita ${ }^{50}$.

c) Aprendizaje sin error. Evitar o disminuir la ocurrencia de errores durante la fase de aprendizaje es un área de gran interés en el campo de rehabilitación de la memoria. Estudios experimentales tanto de laboratorio como en la vida real con pacientes con trastornos del aprendizaje, esquizofrenia y deterioro de memoria por daño cerebral han mostrado que disminuir los errores al mínimo durante la fase de aprendizaje, mejora la adquisición de conocimientos específicos, en relación con el aprendizaje por ensayo y error. Posiblemente los sujetos con amnesia pueden adquirir esta información a través de procesos de aprendizaje implícitos relativamente intactos, pero muy susceptibles a la interferencia causada por errores tempranos, ya que la memoria implícita no puede distinguir la respuesta correcta de una respuesta falsa inadvertida ${ }^{51}$. Cuando la memoria explícita está más o menos deteriorada, como en la enfermedad de Alzheimer, algunos errores producidos durante el aprendizaje son reforzados por la repetición y por ello, la disminución de errores podría ser un decisivo componente de la terapia de memoria (Tabla 6).

d) Estimulación de la memoria procedimental. El aprendizaje de las capacidades sensoriomotoras de los pacientes con 
enfermedad de Alzheimer también ha sido utilizado para el desarrollo de programas de actividades de la vida diaria. El entrenamiento se concentra en los aspectos motores de las actividades diarias más o menos complejas, tales como el aseo personal, preparación de la comida y uso del teléfono. Zanetti y $\mathrm{col}^{52}$ demostraron que los pacientes con enfermedad de Alzheimer leve o moderada, después de tres semanas de entrenamiento, mejoraban significativamente el tiempo empleado en la realización de tareas procedimentales, no sólo de aquellas que habían sido entrenadas, sino también actividades no entrenadas. Estos datos han sido corroborados posteriormente por un estudio controla$\mathrm{do}^{53}$ : once sujetos con enfermedad de $\mathrm{Alz}$ heimer que vivían en la comunidad fueron individualmente entrenados en la realización de 13 actividades de la vida diaria básicas e instrumentadas durante tres semanas consecutivas (una hora diaria durante cinco días a la semana). Se midió el tiempo de ejecución para realizar estas actividades al inicio del programa y cuatro meses después y se comparó con un grupo control de siete pacientes. El grupo de estudio mostró una disminución importante del tiempo utilizado a los cuatro meses de seguimiento, lo que indica un mantenimiento del efecto de la intervención a largo plazo.

\section{Utilización de las ayudas de memoria externas}

Aparte de las estrategias que buscan optimizar los resultados cognoscitivos o hacer aprender nuevos conocimientos explotando las capacidades preservadas, también se puede proponer el empleo de ayudas externas o el acondicionamiento del contexto físico para reducir el impacto de los déficit cognoscitivos en su vida cotidiana. Sin embargo, para que una ayuda sea eficaz el paciente la debe utilizar de forma espontánea.

\section{Ayudas de memoria no electró- nicas}

El uso de ayudas externas como libros de notas, diarios, agendas, es otra estrategia útil en la rehabilitación neuropsicoló- gica. La utilidad de estas ayudas ha sido evaluada en diversos estudios. Hanley y Lusty $^{54}$, en un estudio de caso único, mostraron un efecto positivo en la orientación personal cuando el paciente fue entrenado en el uso de un reloj y un diario. Bourgeois y col ${ }^{55}$ realizaron un estudio controlado en el que confirman la mejoría de la comunicación y de la memoria prospectiva en pacientes con enfermedad de $\mathrm{Alz}$ heimer leve o moderada a través del uso de un libro de memoria. Este contenía dibujos de los parientes y cuidadores, con una descripción de la rutina diaria y sus actividades a través de dibujos y relojes que mostraban el tiempo de su realización. Siete cuidadores fueron entrenados para implementar esta ayuda externa en el momento en el que el paciente exhibiera preguntas repetitivas. La media diaria de verbalizaciones repetitivas disminuyó de 21,9 al inicio del estudio a 11,2 a las doce semanas y a 8,6 a los seis meses de seguimiento. Los cuidadores además referían poder aplicar la intervención en otras conductas no seleccionadas. Es importante tener en cuenta que el éxito de las ayudas de memoria externas depende de la metodología adoptada para enseñar el uso efectivo de las mismas ${ }^{56}$.

\section{Ayudas de memoria electrónicas}

Enseñar a los pacientes a utilizar las ayudas externas tan independientemente como sea posible, sin la dependencia de tener que proporcionar las claves de ayuda por parte de un familiar o cuidador no siempre es aplicable en la vida diaria. Un camino para solucionar este problema puede ser el empleo de agendas electrónicas portátiles y fáciles de manejar, que puedan transmitir vocalmente al paciente, en el momento justo, la información necesaria. La agenda emite, en el momento justo, una señal acústica o alarma vibratoria controlada por un botón central fácil de presionar por el paciente, seguido por el mensaje explicatorio. Estas ayudas han sido utilizadas con éxito en pacientes con amnesia severa no progresiva incrementado su autonomía en la vida diaria sin depender de sus familiares o cuidadores. También ha sido recientemente aplicado en pacientes con enfermedad de Alzhei- 
mer $^{57}$. Cinco pacientes con enfermedad de Alzheimer leve o moderada fueron entrenados en el uso de la agenda electrónica. Se programaron siete tareas prospectivas que debían ser realizadas en horas concretas. La ejecución fue comparada en diferentes días con dos condiciones control donde las mismas acciones eran realizadas por recuerdo libre después de un periodo de entrenamiento o bien a través de la ayuda de un listado escrito. Los resultados mostraron mejores rendimientos en la ejecución en la condición experimental frente a las otras dos (recuerdo libre y facilitado a través de la ayuda escrita).

El uso de ayudas externas no electrónicas todavía no se ha evaluado extensamente, pero puede ser una ayuda importante para los pacientes con enfermedad de Alzheimer en estadios leves con un adecuado entrenamiento en su manejo. También queda por confirmar la validez ecológica de las ayudas electrónicas, pero en un futuro puede ser una herramienta útil en los estadios iniciales de la enfermedad.

\section{CONCLUSIONES}

En esta revisión sólo hemos descrito algunas de las intervenciones diseñadas para mejorar la cognición en los pacientes con demencia. Hay muchas otras dirigidas al resto de ámbitos de intervención: control de los trastornos de conducta, reestructuración ambiental y soporte familiar, quizás más aceptadas y menos discutidas que las intervenciones cognitivas.

En el momento actual y en ausencia de un tratamiento curativo, no cabe duda de que la atención al paciente con demencia requiere un abordaje multidimensional, no teniendo cabida las posturas nihilistas. Las terapias no farmacológicas, en todas sus dimensiones, pretenden disminuir el exceso de discapacidad que la enfermedad de Alzheimer y otras demencias provocan, bien por los propios déficit cognitivos que interfieren en funciones todavía preservadas (síndrome disejecutivo, por ejemplo), bien por los síntomas afectivos y conductuales que deterioran el rendimiento del individuo o bien por la respuesta del entorno (unas veces superprotectora y otras poco estimulante) que impide al enfermo desarrollar todo su potencial.

La estimulación psicocognoscitiva puede ser una herramienta muy útil para ello. Las directrices internacionales para la práctica clínica en el tratamiento de la demencia $^{58}$ recomiendan la aplicación de todas aquellas intervenciones que incrementen la autonomía funcional y mejoren el estado afectivo de los pacientes y de sus cuidadores. No existen todavía evidencias claras (tipo I) sobre el poder de las intervenciones cognitivas. Sin embargo, empieza a haber datos en la literatura de la eficacia de estas técnicas (mejoría en el recuerdo de tareas o información específicas, de la memoria procedimental) y su posible efecto sobre la evolución de la enfermedad (retraso del deterioro).

Por ello, son necesarios más estudios que permitan determinar la eficacia de estas intervenciones y consecuentemente, aconsejar su aplicación de forma generalizada, teniendo en cuenta el despliegue de recursos necesarios.

\section{BIBLIOGRAFÍA}

1. Woods, RT. Non-pharmacologial techiniques. En: Qizilbash N, Schneider LS, Cui E, Tarior P, Brodaty H, Kaye J, Erkinjunyi T. Evidencebased Dementia Practice. Ed: Blackwell Science Ltd. Oxford 2003: 428-446.

2. KiTwood T. Dementia Reconsidered: The person comes first. Open University Press, Buckingjam, 1997.

3. Wilson RS, Mendes de Leon CF, Barnes LL, Schneider JA, BIENias JL, Evans DA et al. Participation in cognitively stimulating activities and risk on incidenT Alzheimer dIsease. JAMA 2002; 287: 742-748.

4. Verghese J, Lipton RB, Katz MJ, Hall CB, DERBY CA, KuSLANSKY G et al. Leisure activities and the risk of dementia in the elderly. N Eng J Med 2003; 348: 2508-2516.

5. FoLSOM JC. Reality Orientation for elderly patient. J Geriatr Psychiatry 1966; 1: 291-307.

6. REEvE W, Ivison D. Use of environmental manipulation and classroom and modified informal reality orientation with institutionalized, confused elderly patients. Age Ageing 1985; 14: 119-121. 
7. Williams R, ReEve W, Ivison D and Kavanagh D. Use of environmental manipulation and modified informal reality orientation with institutionalized, confused elederly subjects: a replication. Age Ageing 1987; 16: 315-318.

8. Brook P, Degun G, MATher M. Reality orientation, a therapy for psychogeriatric patients: a controlled study. Br J Psychiatry 1975; 127: 42-45.

9. Spector A, OrRell M, Davies S, Woods B. Orientación a la realidad para la demencia (Cochrane Review). In: The Cochrane Library. Update Software. Oxford 2000.

10. Woods RT. Reality Orientation and Staff Attention: A controlled Study. Br J Psychiatry 1979; 134: 502-507.

11. HANLEY IG, McGuiRe RJ, Boyd WD. Reality Orientation and Dementia: A controlled trial of two approaches. Br J Psychiatry 1981; 138 : 10-14.

12. Wallis GG. Baldwin M, Higginbotham P. Reality Orientation Therapy: a controlled trial. Br J Med Psychol 1983; 56: 271-277.

13. Baines S, SAXBY P, EHLERT K. Reality Orientation and Reminiscence Therapy: A controlled cross-over study of elderly confused people. Br J Psychiatry 1987; 151: 222-231.

14. Ferrario E, Cappa G, Molaschi M, Rocco M, FABRIS F. REALITY Orientation Therapy in institutionalized elderly patients: preliminary results. Arch Gerontol Geriatr 1991; Suppl 2: 139-142.

15. Gerber GJ. Prince PN, Snider HG, Atchison K, DuboIS L, KILGOUR JA. Group activity and cognitive improvement among patients with Alzheimer's disease. Hospital Community Psychiatry 1991; 42: 843-5.

16. Baldelli MV, Pirani A, Motta M, Abati E, MARIANI E, MANZI V. Effects of Reality Orientation Therapy on patients in the community. Archiv Gerontol Geriatrics 1993; 17: 211-218.

17. Breull V, De Rotrou J, Forette F, Tortrat D, Ganancia-Ganem A, Frambourt A et al. Cognitive stimulation of patients with dementia: preliminary results. Int J Geriatr Psychiatry 1994; 9: 211-217.

18. De Rotrou J. Stimulation et rééducation de la memoire. Gazette Méd 1989; 96: 49-53.

19. Zanetti O, Metitieri T, Bianchetti A, Trabucchi M. Reality orientation therapy for patients with dementia: a longitudinal study. Neurobiol Aging 1998; 19 (Suppl 4): 102-103.

20. Dietch JT, Hewett LJ, JonEs S. Adverse effects of reality orientation. JAGS 1989; 37: 974-976.
21. Powell-Proctor L, Miller E Reality Orientation: A Critical Appraisal. $\mathrm{Br} \mathrm{J}$ Psychiatry 1982; 140: 457-463.

22. BUTLER RN. The life review: an interpretation of reminiscence in the aged. Psychiatry 1963; 26: 65-76.

23. Woods RT, Portnoy S, HeAd D, Jones GMM. In: JoNes \& MEISE, ED. Care Giving in Dementia: Research and Applications. Routledge, 1992.

24. KIERNAT JM. The use of life review activity with confused nursing home residents. Am J Occupational Therapy 1979; 33: 306-10.

25. GoldWASSER AN, AUERBACH SM, HaRkinS SW Cognitive, affective and behavioural effects of reminiscence group therapy on demented elderly. Int J Aging Human Development 1987; 25: 209-22.

26. ORten JD, Allen M, CoOK J. Reminiscence groups with confused nursing centre residentes: an experimental study. Social Work Health Care 1989; 14: 73-86.

27. Spector A, OrRell M, Davies S, Woods RT Terapia de reminiscencia para demencia (Translated Cochrane Review). En: The Cochrane Library. Oxford: Update Software.

28. TÁRRAGA L. Estrategia no farmacológica del deterioro cerebral senil y demencia. Medicine 1994; 6: 44-53.

29. Reisberg B, Franssen EH, Hasan SM, Monteiro I, BOKSAY I, SOUREN LE et al. Retrogenesis: clinical, physiologic and pathologic mechnisms in brain aging, Alzheimer's and other dementing processes. Eur Arch Psychiatry Clin Neurosci 1999; 3: 28-36.

30. Piaget J, Indelher B. Psicología del niño. Ed Morata. Madrid 1975.

31. TÁRRAGA L. El programa de psicoestimulación integral, tratamiento complementario para la enfermedad de Alzheimer. Revista Española de Geriatría y Gerontología 2000; 35 (Supl. 2): 51-64.

32. ERmini-Füngschilling D, Hendriksen C, MeIER D, REGARD M, STÄHELIN $H$. entrenamiento cognitivo en pacientes externos con demencia leve: efectos sobre el estado de ánimo y las funciones cognitivas. En: Fitten J, Frisoni G, Vellas B. Investigación y práctica en la enfermedad de Alzheimer. Glosa ediciones. Barcelona 1998: 221-239.

33. Francés I, Barandiaran M, Marcellán T, InTXAURRONDO B, GuERRERO R. Terapias no farmacológicas en la enfermedad de Alzheimer. Memoria presentada en el Departamento de Salud del Gobierno de Navarra. Pamplona, 2002. 
34. Hoffmann M, Hock C, KüLler A, Muller-Spanh F. Interactive computer-based cognitive training in patients with Alzheimer's disease. J Psychosom Res 1996; 30: 493-501.

35. Franco MA, Orihuela T, Bueno Y, Cid T. Programa Gradior. Programa de evaluación y rehabilitación cognitiva por ordenador. Valladolid: Edintras 2000.

36. Franco MA, BuEno Y. Uso de las nuevas tecnologías como instrumentos de intervención en programas de psicoestimulación. En: Agüera L, Martín M, Cervilla J. Psiquiatría Geriátrica. Ed. Masson. Barcelona 2002: 665-677.

37. ORTIZ T. Teleterapia mediante la televisión para frenar el deterioro cognitivo en la enfermedad de Alzheimer. V Congreso Estatal de Intervención Social. Madrid 1998.

38. Rogers JC, Holm MB, Burgio LD, HSu C, HaRdin JM, McDowell BJ. Excess disability during morning care in nursing home residents with dementia. Int Psychogeriatr 2000; 12: 267282.

39. Butters MA, Soety E, BecKer JT. Memory rehabilitation. In: Handbook of Neuropsichology and Aging, Nusbaum PD (ed.) Plenum Press: New York 1997; 515-527.

40. LIPINSKA B, BÄCKMAN L. Encoding-retrieval interactions in mild Alzheimer's disease: the role of access to categorical information. Brain Cogn 1997; 34: 274-286.

41. MoAYeri SE, CAHILL L, JiN Y, PotKIN SG. Relative sparing of emotionally influenced memory in Alzheimer's disease. Neuroreport 2000; 11: 653-655.

42. Lipinska B, Bäckman L, Mantylä T, VitTANen M. Effectiveness of self-geneated cues in early alzheimer's disease. M Clin Exp Neuropsychol 1994; 16: 809-819.

43. ARKIN SM. Audio-assisted memory training with early Alzheimer's patients. Clin Gerontol 1992; 12: 77-96.

44. JoHnson DL, SMith SD. Effects of familiarity and temporal organization on memory for eventes schemas in aged and Alzheimer subjects: implication for clinical managemente. Alzheimer Dis Assoc Disord 1998; 12: 18-25.

45. DE VREESE LP, NERI M. Ecological impact of combined cognitive training programs (CPT) and drug treatment (ChE-I) in Alzheimer's disease. Int Psychogeriat 1999; 11 (Supl. l): S187.

46. LANDAUER TK, BJORK RA. Optimum rehearsal patterns and name learning. In: Practical Aspects of Memory. Gruneberg MM, Morris
EE, Sykes RN (eds). Academic Press. London 1978: 625-632.

47. Glisky EL, Schacter DL, Tulving E. Learning and retention of computer-related vocabulary in memory-impaired patients: the method of vanishing cues. J Clin Exp Neuropsychol 1986; 8: 292-312.

48. CAMP CJ, BIRD MJ, CHERRY KE. Retrieval strategies as a rehabilitation aid for cognitive loss in pathological aging. In Cognitive Rehabilitation in Old Age. Hill RD, Bäckman L, Stigsdotter Neely A (eds.). Oxford University Press: Oxford 2000: 224248.

49. Davis RN, Massman PJ, Doody RS. Cognitive intervention in Alzheimer's disease: a randomized placebo-controlled study. Alzheimer Dis Assoc Disord 2001; 15: 1-9.

50. De Vreese LP, Neri M, Fioravanti M, Belloi L, ZANETTI $O$. Memory rehabilitation in Alzheimer's disease: a review of progress. Int J Geriatr Psychiatry 2001; 16: 794-809.

51. Wilson BA, BadDELey A, Evans J. Errorless learnin in the reahbilitation of memory impaired people. Neuropsychol Rehabil 1994; 4: 307-326.

52. Zanetti O, BinetTi G, RozZini L, Bianchetti A, TRABUCCHI M. Procedural memory sitmulation in Alzheimer's disease: impact of a training programme. Acta Neurol Scand 1997; 95: 152-157.

53. Zanetti O, Zanieri G, Di Giovanni G, De VReesse LP, Pezzini A, Metitieri T, Trabucchi M. Effectiveness of procedural memory stimulation in mild Alzheimer's disease patients: A controlled study. Neuropsychol Rehabil 2001; 11: 263-272.

54. HANLEY IG, Lusty K. Memory aids in reality orientation: a single-case study. Behav Res Ther 1984; 22: 709-712.

55. Bourgeois MS, Burgio LD, Schulz R, Beach S, PALMER B. Modifying repetitive verbalization of community-dwelling patients with Alzheimer's disease. Gerontologist 1997; 37: 30-39.

56. BIRD M, KINSELLA G. Long-term cued recall of tasks in senile dementia. Psychol Aging 1996; 11: 45-56.

57. Zanetti O, Zanieri G, De VReese LP, Frisoni G, BinetTI G, TRabucchi M. Ug an electronic memory aid with Alzheimer's disease patients. A study of feasibility. 6th International Stockholm/Springfield Symposium on Advances in Alzheimer Therapy. Stockholm, Sweden 2000. 
58. De VReese LP, Neri M, Fioravanti M, Belloi L, ZANETTI $O$. Memory rehabilitation in Alzheimer's disease: a review of progress. International Journal of Geriatric Psychiatry 2001; 16: 794-809.

59. BARANDIARAN M. Programas de psicoestimulación cognitiva en las demencias. En: Manubens JM, Berthier M, Barquero S. Neurología conductual: fundamentos teóricos y prácticos. Ed Pulso S.L. Barcelona 2002: 307-319.

60. Clare L, Wilson Ba, Carter G, Breen K, Goces A, HoDGES JR. Intervening with everyday memory problems in demenita of Alzheimer type: an errorless learning approach. J Clin Exp Neuropsychol 2000; 22: 132-46.

61. Clare L, Wilson Ba, Carter G, Roth I, Hodges JR. Relearning face-name associations in early Alzheimer's disease. Neuropsychology 2002; 16: 538-47.

62. Clare L, Wilson BA, Carter G, Hodges JR. Cognitive rehabilitation as a compnent of early intervention in Alzheimer's disease: a single case study. Aging Ment Health, 2003; 7: 15-21. 